\title{
VON DER LEBENSPHILOSOPHIE ZURÜCK ZUM TRANSZENDENTALEN IDEALISMUS \\ EINE FUNDAMENTALANTHROPOlOGISCHE PERSPEKTIVE \\ AUF DEN THEORIEBEGRIFF DES /LEBENS〈 BEIM SPÄTEREN FICHTE
}

\section{‘Lebenı: ein Begriff jenseits seiner philosophischen Strömung}

Seit die Analytische Philosophie klassischen Zuschnitts ins Stocken geraten und auf der Suche nach neuen Orientierungen ist, wird das systematische Philosophieren von verschiedenen revivals erfasst: revivals philosophischer Programme aus dem Ende des 19. und dem ersten Drittel des 20. Jahrhunderts. Neukantianismus, Hermeneutik, klassischer Pragmatismus, Phänomenologie, Philosophische Anthropologie sollen eine Erweiterung in die Problemperspektiven, Methoden und nicht zuletzt Sachthemen bringen. Das gegenwärtige Philosophieren ist dementsprechend von einer Reihe von Konvergenztendenzen gekennzeichnet. ${ }^{1}$

An diesen Aktualisierungen - Aktualisierungen in >systematischer Absicht - hat die Lebensphilosophie nur recht partiell Teil. Von der Lebensphilosophie als Programm erwartet man sich, den besonderen Fall G. Simmel wohl ausgenommen, nur begrenzt neue Befruchtungen. Entsprechend randständig steht die einstmals große Bewegung in den heutigen Diskussionen. ${ }^{2}$ - Dem scheint eine auffällige Präsenz von Erbschaften >lebensphilosophischen ‘ Denkkontexts befremdend zu kontrastieren. Denn, auf der anderen Seite, die Rede von »Leben« und »lebendig« hat eine hohe Konjunktur in unseren Begrifflichkeiten. Das sind nicht nur die schon terminologisch gewordenen Kompositabildungen, wie »Lebensform«, »Lebensführung«, »Lebenswelt«; oder auch »Lebensverhältnisse «, »Lebenssphären«, »Lebensorientierung«, »Lebenskunst« u. dgl. mehr. Sondern Konjunktur hat auch manche im letzten Grund mit der Perspektive \Leben< verbundene Art der Fragestellung. So die typologischen Verfahren (wie sie ursprünglich aus der klassischen Lebensphilosophie erwachsen waren); die Hervorbringens-Perspektive (die allemal mehr ist als logische Konstituierung), die Phänomene zumal als menschliche Schöpfungen, Unternehmungen oder doch Effekte; die Unterscheidung von Phänomenevolution (oder -emergenz) und (nachträglich-sekundärer) deutender >Rationalisierung ; die Zugehörigkeit zu, Eingebundenheit in getätigte - eben gelebte - Praktiken, in einem weitergespannten Sinne auch: die Rolle einer Sache in Funktionsbewandtnissen; dann die jede Regel durchbrechenden innovativen (oder auch schlicht anarchischen, oder verausgabend-exzessiven) Energien des Individuums; sowie überhaupt, mit der Begrifflichkeit von »Leben« und »lebendig«

1 Verwiesen sei hier nur auf die vielleicht am weitesten fortgeschrittenen Bemühungen dieser Art: die Konvergenz von Analytischer Philosophie und Hermeneutik sowie die Konvergenz von Analytischer Philosophie und Phänomenologie; ferner die Ausweitung des Begriffs des ^Neukantianismus`, so dass bei einigen auch die Gründungsväter der Analytischen Philosophie, zumal Frege und Husserl, darunter zu stehen kommen.

2 Auch bei G. Simmel, der Gegenwärtigkeit Simmels im heutigen Philosophieren, ist es weniger das Lebensphilosophische als solches, als grundlegende, Anderem opponierende theoretische Konzeption, sondern er empfiehlt sich durch seine Themen - deren innovative Breite, seine mit auch >psychologischen und >soziologischen` Reformulierungen - und seine smikrologischen` Analysen. 
festgefahrenen szientifistischen, rationalistischen, gnoseologischen Selbstverständlichkeiten opponieren, sie verflüssigen zu wollen.

$\mathrm{Zu}$ diesem Befund möchte ich Erörterungen auf den Weg bringen, hinter denen eine These in zwei Teilen steht. Erstens, es gibt ein Unabgegoltenes - und Untilgbares, nicht eliminativ zu konvertieren, nicht zu bereinigen -, was die Begrifflichkeit des »Lebens« beinhaltet. Etwas, was sie in die Theoriebildungen hineinbringt. Und zweitens, die klassische Lebensphilosophie, deren theoretisches Programm, hat das Potential der Begrifflichkeit nicht in zureichend unzweideutiger Weise zum Tragen gebracht. Die dazu erforderliche Reflektiertheit hat sich vielmehr in der nachkantischen idealistischen Philosophie entwickelt. Die thematische >Lebensphilosophie dagegen hat die Kategorie des »Lebens «, ihre theoretische Valenz, in Wesentlichem missverstanden. »Leben« ist vor allem ein Platzhalter-Begriff. Oder Stellvertreter-Begriff. Ein Begriff, um ein Problem anzuzeigen bzw. eine Dimension offenzuhalten. ${ }^{3}$ Und daraus schöpft sich auch seine Potenz in Kompositabildungen wie »Lebensform«, »Lebensführung « usw.

Alle drei großen Exponenten des nachkantischen Idealismus, Fichte, Schelling und Hegel, haben in einer bestimmten entscheidenden Phase ihres Philosophierens dem Gedanken des "Lebens« eine prominente Bedeutung gegeben. Was Hegel betrifft, so gehört dies seit den Deutungen von Dilthey und frühem Marcuse zum festen Bewusstsein der Forschung ${ }^{4}$ und ist seitdem vielfach thematisiert worden; in den jüngsten Debatten vor allem um die Problembewandtnisse des Praktischen, d.h. Intersubjektiven (»Anerkennung «, $»$ Liebe«, »Verstehen« kraft wechselseitiger Positionsübernahme, sowie Selbstverwirklichung-und-Arbeit), und der dynamischen Konzeption des Mentalen, d.h. der Logik des Geistigen, bereichert. Bei Schelling sind es, verallgemeinernd gesagt, beim Rekurs auf »Leben « zum einen anti-mechanistische - hinzu prinzipieller: anti-formallogische - Motive, die aus seiner frühen Naturphilosophie, dort verdichtet im Gedanken der »Produktion«, der »natura naturans« und des "Organismus«, ab etwa 1804/06, seit der sog. Freiheits- und Weltalter-Philosophie auf die Grundthemen seines ganzen Systems übergreifen; sowie Motive einer philosophischen Theologie und philosophisch-theologischen Anthropologie - die Persönlichkeit Gottes und die Selbstgestaltung des menschlichen Existenzvollzugs im Angesicht des personalen Gottes-Bezugs, d.h. "Leben« wesentlich als Signum von »Persönlichkeit» (und deren Freiheit anbetrachts der Kausalität der Welt).

Ich werde mich im Folgenden an Fichte halten. Das ist ein in systematischer Hinsicht noch kaum ausgelotetes Feld. Was Fichte zeigt, ist »Leben « als unverzichtbarer Elementarbegriff der Vernunftreflexion. Und im speziellen steht »Leben« bei Fichte für ein konzeptionelles Problem einer Theorie des Mentalen. Dieser Rekurs auf Phänomensachverhalte - Elementarphänomene des Mentalen -, die nur mittels der Kategorie des »Lebens« adäquat in eine Theorie einzubringen sind, weiterzudenken könnte sich denn zu einer fruchtbaren Konvergenz mit heutigen Bemühungen der Philosophie entwickeln.

3 Einige interessante Aktualisierungen, die in dieser Perspektive zu lesen wären, in: R. Elms, K. Köchy, M. Meyer (Hg.), Hermeneutik des Lebens. Potentiale des Lebensbegriffs, Freiburg/München 1999.

4 Vgl. W. Dilthey, Die Jugendgeschichte Hegels, Berlin 1905; H. Marcuse, Hegels Ontologie und die Theorie der Geschichtlichkeit, Frankfurt/M. 1932. Die interpretatorische Problematik, Hegel beidemal je verschieden - seinerseits nahezu zum reinen Lebensphilosophen werden zu lassen, gehört wohl mit zu einem solchen initialen Anstoß. Das ist heute Vorgeschichte. 


\section{Die Konvertibilität von >Sein` und \Leben` am Letztpunkt der Transzendentaltheorie}

Der Gedanke des »Lebens« kommt bei Fichte in dreierlei Zusammenhängen, und entsprechend auch Begriffsbewandtnissen, ins Spiel: als das Problem von Philosophieren bzw. "Wissenschaft« und Leben, d.h. alltäglichem, gewöhnlichem Leben der Menschen (sowie in erweitertem Sinne: dem sozialen Leben, als dem Motivationsboden, Zwecksinn und Bewährungshorizont philosophischer Reflexion); zweitens als Charakteristikum, wo das »Fühlen«, das »Streben«, der »Trieb«-Charakter usw. thematisiert werden, d. h. allgemein die impuls-gebenden Grenz-Erfahrungen, Grenz-Prozesse des erkennenden und handelnden Ich - der Begriff »Leben« also als Signum unseres menschlichen Sinnen-Wesens; und drittens, und das ist das eigentlich Interessante, wo »Leben « zu einer entscheidenden Kategorie in der Grundlegungsargumentation wird. Dies Dritte ist überhaupt erst in der späteren Entwicklung von Fichtes Denken hereingekommen. ${ }^{5}$ Und bedarf ebenfalls noch einmal einer Differenzierung.

Denn seine Charakteristik entfaltet der Gedanke des »Lebens«, und Fichtes Operieren damit, nicht in den letzten und höchsten Grundlegungsargumentationen, sondern sozusagen in den vorletzten. Bei den höchsten, wie sie das Erkenntnistelos - das Reflexionsunternehmen - der Fichte'schen Wissenschaftslehre(n) ausmachen, kommt die Begrifflichkeit von »Leben« und »lebendig« tendenziell nur operativ in Rechnung. ${ }^{6}$ Besondere, exemplarische Bedeutung besitzt dabei eine nochmalige völlige Neukonzeption jenes Programms der Letztgrundlegung aus dem Jahre 1804, die sog. Wissenschaftslehre 1804 (2. Vortrag). ${ }^{7}$ "Leben« kommt dort im Rahmen - am entscheidenden Um-

5 Das Erste fungiert bei ihm, dem hohen Sinn seines Begründungsprogramms gemäß, allenthalben; mengenmäßig aber vor allem in Einleitungen und »populären« Schriften. So bes. in den Grundzügen des gegenwärtigen Zeitalters (1804) - desgleichen den Reden an die deutsche Nation (1808) und der späten Staatslehre (1813) -, seiner geschichtsphilosophischen Perspektive, und in der Schrift, die den Begriff auch explizit im Titel trägt, der Anweisung zum seeligen Leben oder auch die Religionslehre (1806). Das Zweite ist hauptsächlich auf die Werke bzw. Kontexte beschränkt, in denen dies tatsächlich mit dazugehört, nicht im Grundlegungs-Druck zurückgeblieben ist. (In den späteren Wissenschaftslehren tritt es denn zusehends stark zurück.) In beidem gewinnt Fichte unter dem Bewandtnistitel »Leben« tiefe sachliche Einsichten, geht aber, was das Problem und eine Konzeption Leben seinerseits betrifft, nicht übermäßig spezifisch über Maximen, die auch bei anderen philosophischen Ansätzen in Geltung gebracht sind, hinaus; in beidem ist, schon durch die Sache, der Begriffssinn einigermaßen klar, die Bandbreite der relevanten Alternativen nicht übermäßig prägnant. - W. Schrader hat beide Zusammenhänge kombiniert und vom zweiten aus eine Rekonstruktion des ganzen Fichte'schen Reflexions-Unternehmens, als orientiert letztlich an der Problematik der (Einholung der) Realitätsgewissheit, versucht, eine heimliche Basisschicht des Ganzen. Vgl. W. Schrader, Empirisches und absolutes Ich. Zur Geschichte des Begriffs Leben in der Philosophie J. G. Fichtes, Stuttgart 1972. Allenthalben aber gilt, das dreierlei Verschiedene ist bei Fichte auf keinen Fall zu vermengen (das hat auch Schrader nicht getan), schon gar nicht - das war vielmehr eine Folge der Lebensphilosophie - das Dritte mit dem Ersten, dem ungleich Gemeinplätzigen, ja als solchem nahezu Banalen von Wissenschaft-und-Leben.

6 Im vorliegenden Zusammenhang kann es nicht darum zu tun sein, das Auftreten des Gedankens des "Lebens« bei Fichte ausführlich darzustellen; zumal durch das für Fichtes Arbeitsweise charakteristische immer noch einmal neue Ansetzen seiner Texte all die zunächst unterschiedlichen Zitat-Stellen letztlich ungefähr dasselbe - denselben Kreis - an Aussagegehalt beinhalten. Es soll im Folgenden denn auch nicht Fichtes zum Teil höchst spezielle Begriffssprache in allem übernommen, gar nachgezeichnet werden. Insgesamt gilt, dass das Problembewusstsein, das in seinem späten Rekurs auf den Gedanken des "Lebens " verdichtet ist, sich im Verlauf eines mehrfachen fundamentalen Wechsels seiner Grundbegrifflichkeit herauskristallisiert hat.

7 J. G. Fichte, Gesamtausgabe der Bayerischen Akademie der Wissenschaften, Stuttgart $1962 \mathrm{ff}$. (= GA), Bd. II/8. 
schlagspunkt - einer metakonzeptionellen Reflexion über Idealismus und Realismus ${ }^{8}$ zur Sprache; und die Einsicht, die mit der Begrifflichkeit des >Lebens « transportiert, darin formuliert wird, ist, dass sowohl realistische wie idealistische Fragestellungen sich in Substantialisierungen des prädikativen

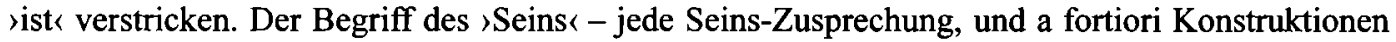
von Wesenheit (»Ansich«) - muss vielmehr an den verbalen Sinn des esse zurückgebunden bleiben; nur in diesem ist die in mehrfacher Bewandtnishinsicht Aktuosität unverkürzt mitenthalten - darum die notwendige Engführung bzw. Konvertibilität von >Seins (- und >Lebens ‘-Begrifflichkeit. Das "reine Sein « ist zu denken als »ein esse in mero actu, so daß beides Sein und Leben, und Leben und Sein durchaus sich durchdringen, in einander aufgehen, und dasselbe sind «. ${ }^{9}$

Die Begrifflichkeit von »Leben« und »lebendig« steht so in jener höchsten Grundlegungsargumentation, um, und zwar am jeweiligen betreffenden thematisierten Problem bzw. der Problemsache, eine bestimmte Struktur anzuzeigen. Es ist namentlich dreierlei, was mit ihr hineingebracht und festgehalten ist: »Leben « überhaupt qua tätig, vollzugshaft, sich prozessierend; dann, dass das Denksubjekt selber dabei ist, ein Gedanke ein vom (freilich nicht privat-individuellen) Subjekt und im Subjekt erzeugter ist - gerade auch jeder (Reflexions-) Gedanke höchster Theoriekonstruktion; und nicht zuletzt die Selbstvorgängigkeit - dass ein Betreffendes immer schon im seinshaften Da seiner Wesensphänomenalität statthat, sich vollzieht, daran weiterbaut, ein esse im Sein, leben im Leben, Reflektieren im Kosmos der Reflexionsgedanken. Gegen schematische PrinzipPrinzipiiertes-Begründungen, vor allem auch >realistischer oder >idealistischer (Theoriecouleur, leitet Fichte daraus denn auch den Strukturtypus der transzendentaltheoretischen Leitphänomene (bzw. sachkonkreter Argumentationsdimensionalitäten) ab, zeigt, wie sie jeweils, in solchem ihrem nichts denn prozessualen Charakter, daraus hervorgehen: zuerst überhaupt das Konzept von Vernunft, und dann >Ich`, Freiheit, Bewusstsein, Realität (d. h. Realitätsgewissheit). ${ }^{10}$ So ist auch Vernunft wesenhaft ihr tätiges Sichäußern (und darin gerade als ent-individualisierendes Geschehnis); Sein ist, verbaliter, im Leben sich vollziehend, ist sein Zum-dastehenden-Sein-Kommen; und alles Konkrete, nämlich in seiner >ontologischen`Zuschreibung, kommt nur in seinem Je-schon zur Ansichtigkeit. Auch was uns Mentalität selber ausmacht, ist mein Sein: Existieren als Vernunft habend, meine Vernünftigkeit lebend, und findend das immer schon vernunftgesetzte Sein. Wir erscheinen uns - verstehen uns - notwendig als Innewerden einer objektiven Vernunft; aber vielmehr, die Vernunft - die Vernunft, welche wir Mentalität als Faktum sind - macht sich vernehmlich: und >Ich/-heit, Freiheit, Bewusstsein usw. als das, wie, d.h. in welchen Strukturen dieser Prozess des Vernünftigkeit-seins sich reflexiv auslegt. Das Wort ist "Vorkommen « - für alles gilt ein letztes nicht weiter ableitbares $» .$. hat statt , und als Teil eines betreffenden umfassenden Statthabens. ${ }^{11}$

Im ganzen ist der Sinn der Rede von »Leben « in der höchsten Grundlegungsargumentation jener Wissenschaftslehre primär der - entsprechend dem verbalen (bzw. prädikativen) esse - adjektivische, das Geltendmachen der nicht hintergehbaren Vollzugshaftigkeit, in der - auch - jede Reflexion immer schon steht, adjektivisch das (umgreifende) Geschehnis, das sich im Denken-Können und Denken-Tun bekundet, bezeichnend: »lebendiges Sein«, »lebendige «, d.h. zu lebende, »Vernunft «, »lebendiger Begriff«, »lebendige Sichkonstruktion« usw. Ungleich tiefer in Bestimmungen, wie dieser Lebensprozess zu denken ist, führt die vorletzte Reflexionsschicht in Fichtes Unternehmen, darunter

8 Worin in dieser Wissenschaftslehre die Durchführung des Grundlegungsprogramms überhaupt in weitem Ausmaß besteht.

9 GA II/8, 228/229.

$10 \mathrm{Vgl} . \mathrm{GA}$ II/8, 414-417.

11 Vgl. bes. den XIV./XV. sowie XXVII./XXVIII. Vortrag; im speziellen GA II/8, 400-403, 410-415, $240 \mathrm{f}$. (»Vorkommen«). 
bes. eine späte Vorlesung vom Winter 1810/11 über Die Thatsachen des Bewußtseyns. ${ }^{12}$ Im Unterschied zur letzten, nach reinen Prinzipien suchenden Grundlegung bleibt ihre Thematisierung - der Thematisierungsfokus, aber nicht die Weise der Argumentation! - im Bewusstseins-Verhältnis, in der Phänomenalität des >etwas ist/wird bewusst<. Alles Inhaltliche, Sachdistinkte ist - erfährt seine Bestimmtheit - uns im Bewusstsein: und die philosophische Reflexion bleibt hier methodisch gezielt an dies angebunden, wie es zunächst einmal auch die Wissens- und Handlungs-Welt alles gewöhnlichen, natürlichen Bewusstseins ausmacht. Doch zielt die Argumentation durchgehend darauf ab - nämlich unter Rekurs auf »Leben« -, dabei genau nicht schon eine bestimmte einfache Formstruktur von 'Bewusstsein ‘ anzusetzen, d.h. unversehens mit zu unterstellen, ${ }^{13}$ ein einfaches Subjekt-Objekt-, Form-Inhalt- oder sonstwie Repräsentations-, gar Bühnen-Modell.

12 GA II/12, 23-136. Drei Semester lang, Winter 1810/11, Sommer 1811, Winter 1811/12, hat Fichte dies im Bau seiner Vorlesungen als Hinführung zum Standpunkt der höchsten Reflexion der >Wissenschaftslehre` vorgetragen; durch vielfältige Hörer, unter Anderem auch Schopenhauer, sowie die Publikation jenes Kurses von 1810/11 (separat 1817, und dann im Rahmen der Sämmtlichen Werke 1845/46) war die Konzeption in den Diskussionen der Zeit prinzipiell bekannt. - Ab 1812 hat Fichte die Hinführung dann zweisträngig entworfen, als zusammen mit einer Transzendentalen Logik- logischen Vorklärungen der Struktur dessen, was Denken ist -, welche dabei ebenfalls auf den Gedanken des »Lebens« rekurriert. Das hat aber genau beides hier nicht mehr wirklich tiefer argumentieren lassen. Die Transzendentale Logik und ihre Frage hatte die grundlegungstheoretische Bedeutung des »Lebens« gewissermaßen absorbiert. (Vgl. denn auch die späte, in ihrer Argumentation ungleich weniger weit ausgreifende Version der Thatsachen des Bewußtseyns vom Anfang 1813, in Vorb. für GA IL/15 [einstweilen: Fichtes Werke, hg. v. I. H. Fichte, ND Berlin 1971 (= SW), Bd. IX, 401-574]; einen Sonderfall in der - nämlich relativ breiten - Argumentation mit »Leben« stellt die Version der Wissenschaftslehre vom Anfang 1812 dar, die bezeichnenderweise in ausgesprochenen Worten ihre Reflexion an die der Thatsachen des Bewußtseyns heranrückt, vgl. GA II/13, 110.) Was die Argumentationsweise betrifft, so zeigt die Transzendentale Logik beim Fungieren des Begriffs des »Lebens « (wenngleich hier weitgehend in substantivischer Rede) große Parallelen mit der Wissenschaftslehre 1804 (2. Vortrag). Vielleicht muss man sogar sagen, dass deren Argumentation vielmehr vereinseitigt ist und vom dortigen (dreifachen) Bedeutungsspektrum vor allem bloß »Leben« qua tätig und qua >... ist hervorgebracht " geltend gemacht ist. Vgl. zum Begriff des "Lebens" in der Transzendentalen Logik die Darstellung von A. Bertinetto, ")Sehen ist Reflex des Lebens . Bild, Leben und Sehen als Grundbegriffe der transzendentalen Logik Fichtes«, in: E. Fuchs, M. Ivaldo, G. Moretto (Hg.), Der transzendentalphilosophische Zugang zur Wirklichkeit, Stuttgart 2001, 269-306.

13 Das beträfe vor allem zweierlei traditionelle Erbschaften von Orientierungen, die immer wieder die Ursache unbefragter Generalvoraussetzungen waren: die Terminologie des Sehens, die aus dem Bereich des Visuellen geschöpfte Begrifflichkeit, die Metaphorik des Räumlichen - Bewusstsein-haben-von ... als Etwas-sehen (dieser Rede bleibt bekanntlich auch Fichte verpflichtet, trennt sich aber in seiner späteren Entwicklung in immer radikaleren Wendungen von den darin unterschwellig vorausgesetzten logischen Sachverhältnissen); und ebenso das Modell - das Wissenschaftsparadigma - des Mathematischen (sowie modern: Kybernetischen), nämlich Bewusstsein zu denken als - eben vermutlich komplexen - Funktions-Operator in Bezug auf durch den sensus gegebene darin eingesetzte Daten-Werte. Hinzu käme, als Drittes, die neuzeitliche 'psychologische< Theorieweise - Bewusstsein als ein Gefüge von >Vermögen`, und dass ein substrathaftes Menschen-ens diese `haber. Eine >Vermögen-haben<-Konzeption unterliegt aber allemal einer prekären eigenen Logik ihres Argumentierens; sie würde entweder nur auflisten nämlich was sich klassifikationstauglich finden lässt - oder hierarchisierte die angesetzten >Vermögen', leitete sie aus einem (nach formalen Kriterien ausgezeichneten) behaupteten höchsten ab, sukzessive abwärtssteigend und ausdifferenzierend. 


\section{Die Wirklichkeit des Bewusstseins und die »Thatsachen« seiner Bedeutungs-Welt}

Die Grundeinsicht jener Vorlesungen ist, dass Bewusstsein vielmehr, sowohl als natürliche Bewusstseinswelt und deren Gewissheitsbekundungen wie auch als >mentalistisches` philosophisches Programm, methodisch gerade zugleich ein Problem darstellt. Die leitende Überlegung der Vorlesungen ist: dass der Kosmos unserer menschlichen Intelligenz durch mehrerlei verschiedene »Thatsachen« ausgezeichnet, oder genauer aufgespannt, ist - Fakta von Was-wirklich und des Bewusstheitsvermögens selbst, die aber gleichsam im blinden Fleck unseres Bewusstseins (bzw.: jeweiligen blinden Flecken seines mehrschichtigen Prozesses) liegen und die (ihm, als sich vollziehend) auch solch Selbstverständlichkeiten scheinen, dass ein Zweifel daran gar nicht in den Blick tritt. Ein mit Bewusstsein begabtes Wesen ist eine komplexe, vielschichtige Struktur seiner (modern gesprochen) mentalen Repräsentationen; der Kosmos unseres menschlichen Welt- und Selbstverständnisses, in dem wir alle uns alltäglich natürlicherweise bewegen - und über den wir uns auch gegenseitig verstehen bzw. verständlich machen -, ist genau besehen von einer Reihe von - ganz unbewusst-selbstverständlichen - Voraussetzungen konstituiert. Sie betreffen, so die Entfaltung der Fichte'schen Vorlesungen, sämtliche Dimensionen unserer Bewusstheit - die theoretisch-kognitive, d.h. unsere Objekte-Vorstellungen, die praktische unseres Handlungsbewusstseins, d. h. Wollens- wie Interventions-Vorstellungen, die Dimension normativer Gültigkeiten, konkret moralisch-sittlicher Reglementierung, und schließlich die Dimension eines Religiösen.

Es sind, und in jeder dieser Dimensionen spezifische, aufschichtend hinzukommende, Hintergrund-Voraussetzungen logischer, struktureller, materialer sowie nicht zuletzt begrifflich-semantischer Art. Innerhalb unserer Intelligenz stehen sie, aber auch das nur bei philosophisch-reflexivem Aufmerken, als: sich findende Phänomenalitäts-Formen, implikative Bedingungen der Phänomengewahrung, Strukturgegebenheiten des Heraustretens eines (betreffenden) Phänomens. Sie aufzudecken - in dieser Hinsicht die transzendental-apriorischen Elemente aller unmittelbaren Phänomenalität, Phänomenalität des Kognitiven, Handlungspraktischen, Normativen und >Religiösen ‘ - steht als Wissenschaftsaufgabe einer höheren Empirie, ${ }^{14}$ ihre »Thatsachen« der Bewusstmachungs-Fähigkeit, des Bewusstseinsprozesses liegen allen anderen gegenständlichen Tatsächlichkeiten voraus. Die normalen Tatsachen - Wissens-Sachverhalte, Wollens-Zwecke und Zustands-Gefühltes -, die sich im >Ist! « unserer Urteile bekunden, ${ }^{15}$ sind nur das (zurückbleibende) Ergebnis gewisser apriorischer Prozesse und deren als Tatsachen-des-Bewusstseins stehender Schematisierungen: der Tatsachen der Weise - in welcher Sachschematisierung -, wie mir die Tatsächlichkeiten meines Urteilens werden. Wo die letzte Grundlegung, wie sie die Wissenschaftslehre 1804 (2. Vortrag) exemplifiziert, aus dem Gedanken des »Lebens«, dem Argumentationsrekurs auf »Leben«, maximal gewisse prinzipielle Schichten metatheoretischer Art, Schichtungen von Denkmodi und Theoriebegrifflichkeiten, >Instanzen` der Selbsterscheinung (>Ich<-heit usw.) herauszuholen vermag, zeigen sich hier materiale Sachstrukturen, und in ihrem logischen, sukzessive aufbauenden Zusammenhang. Fichtes scheinbar bescheidene Darlegungen in den Thatsachen des Bewußtseyns, die zuweilen fast als einzelne Stücke oder Stichlinien einer Art Selbstbeobachtung daherkommen, stellen in Wirklichkeit in hoch anspruchsvolles Programm dar.

Die allgemeine Weise dieser Voraussetzungen wäre zu fassen als: Gegenständlichkeit, genauer Form von Gegenständlichkeit. Die »Thatsachen des Bewußtseyns« konstituieren verschiedene Modi

14 Deutlich ausgesprochen in der allgemeinen Einleitung in die Philosophie vom Winter 1810/11, als Fichte dort den Übergang zu den Thatsachen des Bewußtseyns vorbereitet: vgl. GA IV/4, 24-29.

15 Und, wie Fichte hinzufugt, auch die Tatsachen, welche das Gegenstandsfeld, den Erkenntnisrahmen der anderen Wissenschaften ausmachen; nicht zuletzt die formalen höheren einer >Psychologie oder >Anthropologie ‘ des Mentalen, in ihren Aussagen über die Tatsachen unserer `Vermögen ‘ - welches ohnehin hochgradige sekundäre Abstraktionen sind. 
von Gegenständen (mir-Gegenständen) - und in diesem ist im Sinne Fichtes sowohl das »Gegen-«, das (relationale) Gegenüber-zu-einem-Bewusstheitspol, zu hören wie auch das »Stehen«, die Festigkeit eines sist! ‘ und ’gibt! ‘ »Leben « nun ist, hier in dieser - spezifisch dieser vorletzten - Grundlegungsargumentation, die vom späteren Fichte eingebrachte Kategorie, um in der Allgegenwärtigkeit des Gegenständlichen das zugleich Agierende, Formende solchen Ins-Gegenüber-Bringens in den Blick - und dann in Argumentation - zu heben. »Leben« ist die Kategorie, in der sich, in Abhebung vom allseitigen - vorauszusetzenden, und schon immer vorausgesetzten - „Stehen « der Gegenstände, der Vollzug, das Geschehen des Bewusstseins-haben-von ..., der Prozess der Bewusst-Machung sowie der Selbstmodifkation (Kritikoffenheit) des Mehr- und Besser-bewusst-haben-Wollens, ins Denken hebt. Denn: alles faktische Bewusstsein ist verflochten mit seinen Gegenständen - ist eingenommen von dem, wovon es, je zuständlich, Bewusstsein hat. Bewusstsein ist: sein je Zustand des Bewusst-Gewordenen, Bewusst-Gemachten. Seine Prozessualität und Spontaneitätsfähigkeit ist zunächst die Befangenheit im Bewussten, dessen Objektivitäts-Da und deren zugehöriger >Ontologie wie >Gesetzlichkeiten<. Sein Eigenes ist sein Fortströmen, Immer-Neu, ${ }^{16}$ dessen es aber unmittelbar niemals zugleich ansichtig wird. Das Bewusstsein, in seiner Lebendigkeit, hat nicht sich, sondern sein (von ihm selber generiertes) Wovon. Selbst für unsere menschliche Rationalität gilt, dass das Bewusstsein, nämlich unser individuelles Bewusstsein und sein Denken, zunächst, d. $h$. in seinen alltagswirklichen Formen als »von der Vernunft besessen « lebt. ${ }^{17}$

\section{Grundlegung einer Hermeneutik des lebendigen Selbst}

Das Faktum der uns auszeichnenden Bewusstheit, in ihrem Lebens-Prozess, ist darum aber auch prinzipiell in keine einfache Theorie zu bringen. In wenigstens vier fundamentalen Hinsichten zeigt es sich in einer unweigerlichen logischen Nicht-Einheit, in der Zusammengehörigkeit von Seiten, deren Sowohl-als-auch und wechselseitiges Aufeinanderverweisen bzw. -verwiesenheit lediglich als Faktum - Faktum des Phänomens des Mentalen - konstatiert und in seiner inwendigen Logik hermeneutisch expliziert werden kann. Welches aber denkerisch nicht auf ein Eines zurückgeführt werden kann, das die Polaritäten, aus denen sich das Phänomenale aufbaut, erklärte, d.h. aus sich ableitete.

- Sobald in Reflexion gebracht, steht, erstens, das Sowohl von Schema des Lebendigseins und Zugleich von irreduzibler, faktischer Vielheit, nämlich Vielheit der faktisch pluralen Exemplare davon - als die Gestaltungen ihrer jeweiligen Individualität und an je raum-zeitlich indexikalischer (leiblicher) Situierung in der (umgreifenden) ,Wirklichkeit.${ }^{18}$

- Dann, dass am Prozess des Bewusstseins - der Bewusstwerdung, Bewusstmachung - nicht die eine Seite des Verhältnisses als irgend fest angesetzt werden kann, erst recht nicht in einer fundamentalen >realistischen ( oder >idealistischen « Metaphysik, d.h. Ableitungsontologie, sondern nur als relationale Polarisierung zweier, eines Gegenstehend-Objektiven (mir-gegenüber gewahrbar) und einer Ichheit-Nichtweltlichkeit, begriffen werden kann, als Polarisierung eines relationalen InsGegenüber-Kommens bzw. -Bringens; sowie dass der Bewusstseinsprozess deshalb auch als solcher nicht einer ist, ein genereller actus oder Mechanismus von eben: Repräsentationen-Haben o. ä., sondern sich in mehrerlei Weisen seiner - ineinanderspielend, sich aufschichtend - manifestiert und auch mit mehrerlei jeweiligen Wirklichkeits->Gegenständlichkeiten`.

16 Fichte macht die ganze Metaphorik von Strömen, Ausströmen, Fortfließen, Verfließen und dergleichen dienstbar. Vgl. etwa GA II/12, 32, 34, 53; auch SW IX, 70, 64 f.

17 GA IV/12, 71.

18 Fichte, um diesen harten Übergang von der Einheit ins Zugleich der Vielheit zu bezeichnen, spricht hier terminologisch von $»$ Spaltung . 
- Drittens die Nicht-Einheit, die mit der Sprachlichkeit bzw. Begrifflichkeit allen Denkens zusammenhängt, ihretwegen nicht zu hinterschreiten oder zu tilgen ist - der `semantische〈 Spalt zwischen begrifflichem Bild (sowie sprachlichem Titel dafür, Titelwort auch für die Verständigung und überhaupt die Urteils-Form des Sprechens) und gemeinter konkreter Sache, Sachvorkommnis.

- Und schließlich die Nicht-Einheit des Sich-hinterher, die Differenz - sozusagen die shermeneutische (Differenz - von je Vor-sich-selber-Dastehen, in Evidenz des Präsentischen, und nach-folgendem Innewerden des Generierungsprozesses dabei, zumal des Identitätspols seiner Quellung. ${ }^{19}$

Das Geschehen des Bewusstseins als »Lebens«-Geschehen zu zeigen fungiert, um, in dieser mehrfachen Hinsicht, das jeweilige Nicht-Eine auseinander- und zugleich zusammenzuhalten: damit die Tatsache des Mentalen nicht zerfasert in eine Vielheit unkoordinierter sprivater ‘ Bewusstseins-Welten; nicht in eine Vielheit nebeneinanderstehender intellektueller resp. psychologischer Instanzen ( Vermögen $)$ bzw. Sachqualitäten (und damit die Verlockungen von sowohl idealistischen wie >realistischen ( Erklärungen abgehalten sind); damit, drittens, die Möglichkeit von >objektiven « geteilten - Bedeutungen begreifbar wird; und damit das Ineinander von Konstitution (Genese) und Geltung begreifbar wird, überhaupt die Möglichkeit der Selbsterhellung. ${ }^{20}$ In all diesen Punkten ist das höchste Wort möglicher Theorie das >... nur zusammen`.

\section{Generierung von Selbstbezeichnungen der Bewusstheits-Relation}

Eine komplementäre fundamentale Einsicht Fichtes in jener vorletzten Reflexionsschicht seiner Grundlegungsargumentation schließt sich dem, in ihrer Tragweite in seinen Vorlesungen freilich fast versteckt, unmittelbar an. Denn schlechterdings aufgegangen in seinen (je zustăndlichen) Gegenständlichkeiten, benommen von seinem Wovon, gäbe es überhaupt kein eigentliches, gar individuelles Bewusstsein, wäre der Kosmos des Mentalen, vollends in seinen shöheren، Geltungs-Gehalten, nicht begreiflich. Es gäbe nur das Fortwälzen von Perzeptions-Daten - dumpfe sensations, in einem Immer-weiter -, von Begehrungen und von Zustands-Empfindungen; selbst die Funktionen des Intelligiblen wären nur das Agieren in/nach Vernunft-Regeln, habitusartige Weisen des Wie - der mentale Fokus benommen von diesem eigenen Wie -, aber eben kein menschliches Bewusstsein.

Bewusstsein, als das Immer-schon-im-Bewusstsein, muss, um mehr zu sein als Speicher- (d.h. Reproduktions-) und operative `Intelligenz‘, mehr auch als die bloße Flächigkeit des (gegenüberstehend) Bewussten, in diesem seinem Kosmos stets auch Formen und Denk-Bilder des Ent-Flechtens, des In-Distanz-Bringens zugleich mitproduzieren. Fichtes Nachweis zielt denn darauf ab zu zeigen: dass zu Bewusstsein transzendental unabdingbar zugleich auch, im Prozess der Bewusst-Werdung, Bewusst-Machung mit hervorgehend, nämlich an den konstituierten Objektivitäten - im Gegenüber zu diesem Gegenüber -, (verfügbare) Muster der Selbstbezeichnung, und Muster der Thematisierung der Bewusstseins-Relation, gehören. Bewusstsein hat seine >Welt zunächst an seinen nichtichlichen Sachtatsachen, in der Gegenständlichkeit des sich weiß ...<, >empfinde ...<, >begehre ...८,

19 In all dem zeigt sich, dass ein »Denken der Differenz« gerade in Fichte sein großes Vorbild hätte. Es ist eine der großen - und blockierenden - denkgeschichtlichen Absonderlichkeiten des 20. Jahrhunderts, dass man, gestützt auf Fichtes frühe Rede vom »Ich«, gesehen im Blick von Husserls (und Sartres) transzendentalem Ego, Differenz und Nichtidentität gerade gegen Fichte meinte in Aufstellung bringen zu müssen.

$20 \mathrm{Hinzu}$, gewissermaßen als fünftes, die Nicht-Einheit, die auch die meisten anderen Theorien als Problem gesehen haben: die Nicht-Einheit, die mit der Je-Zuständlichkeit des Bewusstseins zusammenhängt, die Frage von Kontinuum-und-Diskretheit - wo »Leben« für das steht, was sich uns metaphorisch als >Bewusstseinsstrom « darstellt, d. h. damit dieser sich nicht in Einzelpunkte auflöst. Und was mit einer konzeptionell >lebensphilosophischen Theorie gerade verdeckt - verharmlost - ist. 
sunterstelle mich einer Geltung von ...<, >bin meiner als sinn-zugehörig zu ... gewiss (zuletzt zu dem Absoluten allen Sinns)<, und versteht sich aus diesen seinen je dastehenden Tatsachen, von ihnen her; aber genau diese Tatsachen, ihr Gespinst, sind schon je durchsetzt mit höheren Deutungstatsachen - in ihnen stehen für das Bewusstsein, im allseitigen Kosmos des Bewussten, der Gegenständlichkeiten, symbolisch-stellvertretende Vorstellungen seiner selbst, operativ handhabbar und in Urteilen zu platzieren. In den einzelnen Bewusstseins-Setzungen, die unser Bewusstseinsleben ausmachen, ist Bewusstsein darum - der Bewusstwerdungs-, Bewusstmachungsprozess - wesentlich immer auch eine >Theories: ist, an den durch es konstituierten Gegenständlichkeiten, den Erfahrungen mit dem Gewordenen abgeformt, >Theorie « über zuerst >was es gibt`, dann, dagegen herausprofiliert, über sich, seine Seinsweise, ontologischen Status, sowie >Theorie` über die Repräsentations-Relation als solche, die Handlungsmächtigkeit von Intentionen, das Gültigkeits-Sein von Normierungen, den Horizont des absoluten Sinns (und der Versöhntheit meines Endlichen und Einzelheit darin).

In der hochkomplexen Konstitution dessen, was das nur scheinbar einfache Faktum des Bewusstseins darstellt, werden unserer Bewusstseinskompetenz, dem Kosmos, worin sie sich bewegt und das einzelne Gegenständlichwerdende platziert, so sukzessive Momente seines eigenen Denkens ${ }^{21}$ immanent, in Gestalt von Gedanken, Gedanken-Figuren des intelligiblen Umgehens-mit ... werden ansichtig und prädizierbar. Des genaueren, wie Fichte darlegt, in mehreren Bögen bzw. Schleifen: wie mir, per Vorstellungs-Schemata, überhaupt (wahrgenommene) `Dinge stehendes Sach-Etwas, substratartig, mit attributierbaren sihre<-Eigenschaften und (wechselnden) >ihre`-Zustände; wie überhaupt die Vorstellung (oder Deutungs-Figur) eines eigenen (mentalen) Vermögens, z. B. schon, mir selber das Vermögen Vorstellen, Vorstellen-Können zuzuschreiben, oder dann generell mich selber als >den (Bewussten zu sehen, der Bewusstseinsgehalte 'hat<, der als deren zeitliche Folge seine Mentalität lebt; die Objektivität von erkannten Kausalitäten (KausalitätsBezügen); die Vorstellungs-Erfassung einer Eigenkontinuität meines Bewusstseinskosmos im sich erinnere mich , Deutung des Gegenständlich-Präsenten als sozusagen innermentale Erinnerung; ${ }^{22}$ dass Empfundenes als Empfindungen-von ... (eines Nicht-Mein) dastehen und (zuständliche) Diskrepanzen von Empfindungskomplex und wahrnehmbaren (als Wahrnehmung interpretierbaren) Ding-Sachverhalten als Selbstempfindung und dann Trieb-gegen ..., Verlangen-nach ..., Agierenzum-Zweck-von ...; darin die Vorstellungs-Ansichtigkeit eigener Freiheit - Freiheitsgrade des Freiseins-von ...; die Vorstellungs-Gegenständlichkeit von smein Körperく, als des Bildes von meiner Wirksamkeitsfähigkeit; das Deutungs-Bild von Mentales-sin(-der-Welt, d. h. ich als nur eine, nicht die ganze, ich als nur individuelle mentale Wirklichkeit und (mögliche) andere Mentalitäts-Wesen außerhalb meiner, Bewusstseinswirklichkeiten außer der meinen; und Anderes mehr. Allgemein ist es die sukzessive Aufschichtung von Polarisierungen - von Verständigungs-Formen des Meinigen und Nicht-Meinigen (und in Letzterem wiederum das Auseinanderbringen von Weltwirklichkeit, anderem Mentalen/Bewusstseinskosmos, bewusstseinsgewirkte Schöpfungen, Gemeinsamgültigkeit von Normierungen usw.), und relativ spät erst mit spezifischer >Ich/-Bedeutung.

21 So Fichtes Begriff hier (zugleich eine Reformulierung des Problems des Apriorischen vom »Denken « her) - seine ganze Theorie mithin auch, unter diesem Verhältnis allgemein zu fassen, als eine Theorie von Leben-und-Denken (vgl. offen bezeichnet GA II/12, $107 \mathrm{f}$., $36 \mathrm{f}$.). Dies sowie die damit zusammenhängende Fichte'sche Theorie der »Bilder« - das stets »Bild«-mäßige all unseres Etwas-Erfassens - ist hier nicht genauer zu verfolgen und zu differenzieren. Verwiesen sei auf meinen Aufsatz »Die Tatsachen der Tathandlung. Über Fichtes implizite Auseinandersetzung mit den Anfängen einer santhropologischen Depotenzierung der Vernunft« (dort bes. der Abschnitt `Das Da des Ich`), in: G. Zöller (Hg.), Grundbegriffe in Fichtes Spätwerk, Amsterdam/New York 2009, 79-128.

$22 \mathrm{Vgl}$. GA II/12, $48 \mathrm{ff}$. 


\section{Bilder der Freiheit}

Diese grundlegungstheoretische Beziehung von Leben und Bewusstsein gibt nun auch die santhropologische` Bewandtnis der Fichte'schen Thematisierungen, >anthropologische` Bewandtnis dieser Theorieweise. Alle drei eingangs bezeichneten großen Zusammenhänge, in denen der Gedanke des "Lebens« bei Fichte fungiert: das Verhältnis von Wissenschaft-und-Leben, dann in Bezug auf uns Menschen als »Sinnen«-Wesen, sowie hier als zentrale Kategorie in der Theoriegrundlegung, sind sichtlich auch anthropologisch zu verstehen, haben eine entscheidende anthropologische Bedeutung und Impetus ihres solchen Denkens. Bei jenem dritten, wie es beim späteren Fichte heraustritt und am deutlichsten in den Thatsachen des Bewußtseyns (zumal der Darlegung von 1810/11) ausgestaltet ist, ist es eine fundamentalanthropologische Bewandtnis: welch komplexe Aufgabe es ist, das Vorkommen eines Lebe-Wesens mit »Bewußtseyn« (menschlichem Bewusstsein) adäquat zu begreifen; und wie alle weiteren klassischen, vor allem auch idealistischen sowie reflexions- und rationalitätstheoretischen Fragen daran zurïckzubinden sind.

Fichte zeigt, wie dieses Wesen, das, und in seinem ontischen Vorkommnis als je Einzeln-Individuell-Endliches sowie Situiertes, seine Bewusstheit ist, durch Bilder - zuhandene Deutungs-Schemata - von seiner Lebendigkeit die Verschmelzung mit seinen Je-Gegenständlichkeiten aufbricht, gegen die Massigkeit der Bewusstseins-Gegenstände sich zu einem Eigenen wird, und dies in fortschreitendem, sich aufschichtendem Selbstkonstituierungsprozess. Ihm werden, emergenterweise in seinem Vollzug, ${ }^{23}$ operative Schemata der Aufschließung seiner Perzeptions- und EmpfindungsMassen - und daran, an der solch »verstandenen « Welt, im Negativ sich herausprofilierend, gewisse Bilder seiner eigenen Lebendigkeit, seiner eigenen Anteiligkeit dabei. ${ }^{24}$ In ihnen wird ihm symbolisch-stellvertretend das - genauer: ein, d.h. ein bestimmtes - Eigene shinter dem allenthalben natürlichen Objektivismus zugänglich, und ergreifbar. Indem es so sich etwas zuzuschreiben antritt, Zuschreibung von Prozess-Momenten an sich (qua >Vermögen`, >Akte〈, intern rückbezügliche `Reflexionen< usw.), werden wiederum die Inhalte modalisiert (১was ich da eigentlich bewusst-habe،, und wie); Gehalte rücken, intellektuellerweise, in Distanz, werden thematisierbar, nämlich jeweilig und Stufe um Stufe. Dadurch auch erst wird überhaupt die inwendige logische Normativität, als essentiell für das Faktum Bewusstsein, verstehbar - die Korrektur-Fähigkeit (১sich irren< etc.), das Zusammenbringen, Ineinanderspiegeln verschiedener Aspekte sdesselben, , die Eigenkriterien des Wahren und Zweckhaften und Zu-Tuenden, die Verhaltensflexibilität, Differenzierung von Situations- qua Agierens-Klassen, u. A. m.; desgleichen erst verstehbar die Könnens-Gewissheiten, die imaginierten Gegebenheiten, die modifizierten Szenarios, das Hypothetisch-Gesetzte, überhaupt das Kontrafaktische - generell gesagt: das zum Faktum Bewusstsein gehörende >Könnte〈, das eine Bewusstseins-Welt untilgbar mit ausmachende Sein- oder Tun-২Könnte, die Möglichkeits-Horizonte.

Das natürliche Bewusstsein, der >Mechanismus` des natürlichen Bewusstseins, ist - faktisch eben das (diese so bestimmten) Polarisierungen-Haben, Zuschreibungen-, Muster-, Bilder-, Deutungen-Haben. Das natürliche Bewusstsein: unsere Wahrnehmens- und Denk-Weise, unsere Wollens-Bildungen, unsere Selbstnormierung, Zueigenmachen von Gültigkeits-Anmutungen usw., ist diese Logik - das Faktum des Vorkommnisses eines solchen Prozessgefüges und in je einem Ein-

23 Fichte spricht von »sich einstellen[...] «, »tritt [...] ein« u. dgl.; vgl. GA II/12, 45, 36.

24 So korrigiert Fichte damals in einer bemerkenswerten Weise - deren Radikalität sich äußerlich kaum zu erkennen gibt - denn seine eigene frühere Theorie in der Grundlage der gesammten Wissenschaftslehre von 1794/95, deren ganzen Ansatzpunkt: "Das Ich setzt sich selbst, ist nicht wahr. Wahr ist: es ist Bild eines Sichsetzens « (SW IX, 217; vgl. auch LX, 484 f., 87!). - Ein kleiner Vorschein auf die spätere Konzeption, aber dann nicht weiter verfolgt, blitzt in vier Seiten Einleitungspassage im Grundriss des Eigenthümlichen der Wissenschaftslehre in Rücksicht auf das theoretische Vermögen von 1795 auf: GA I/3, 143-146. 
zelwesen. Auch hierfür gilt das snur zusammen<. Unser Bewusstsein ist so verfasst; anders gibt es, als Faktum, Bewusstsein nicht. Seine Faktizität ist nur, in reflexiver Hermeneutik, aufhellbar, Zusammenhänge verständlichmachend rekonstruierbar, aber bei Gewissem ohn weiteres Warum.

Der Gedanke des »Lebens « ist dabei Index für den Prozess der Selbstbestimmung qua Selbst-Ausgrenzung aus der Welt; am Gedanken des »Lebens« wird Bewusstsein - wird sich - als dieser Prozess der Selbst-Aussonderung aus der - sseiner - Welt begreiflich. Lebendig sein Sein seiend, und das >Ist! ‘ seiner Prädizierungen setzend, setzend zu einer dastehend-gegenüberstehenden Realität, zeigt Bewusstsein sich als der Aufbau - und die Aufrechterhaltung - seines Kosmos von Substantialitäten, Kausalitäten, Notwendigkeiten und Möglichem, der Aufbau abgerungen der Macht (je einnehmenden Präsenz) des von ihm selbst zur Geltung erhobenen ‘äußeren، Wovon. Es ist ein Aufbau im Begreifen des Äußeren, in aufschichtend differenzierteren Kategorien und Schematisierungen, wie zugleich gewissermaßen >nach innen<, zu Vorstellungs-»Bildern« von sich, von seiner das zum Stehen Gekommene generierenden Lebendigkeit. ${ }^{25}$ Und dies dann sedimentiert und auf Dauer gestellt in der Vorstruktur eines bestimmten $»$ Anschauung[s]=Systems $«,{ }^{26}$ geronnen zu einer festen Weise, festen Schemata der Welt- wie Selbst-Gewahrung, Begrifflichkeiten der Welt- wie Selbst-Bezeichnung, und zu einer im Wandel seines andrängenden Wovon bleibend-stabilen sinneren` Eigenstruktur.

Im ganzen ist es der große Prozess der Befreiung des Bewusstseins, Befreiung von der Macht seiner eigenen unablässig je schon gewordenen Vorstellungen, seine Befreiung zu sich. Die Selbstanschauung des Lebens $»$ [bricht sich] nothwendig in [diese zuhandenen schematisierenden] Bilder, [...] [nämlich] um jenseit aller [präsentischen] Anschauung sich auch noch denken zu können«. Das »Leben [des Bewußtseyns] beginnt in einer gewissen Gebundenheit seiner Freiheit. Sein Fortgang und Lebenslauf besteht darin, daß es sich aus dieser Gebundenheit befreie, wodurch es wohl einer andern, aber mindern Gebundenheit anheim fallen dürfte, aus der es wiederum sich zu befreien hat, u. s. w. [So ist] sein Lebenslauf [...] eine fortgehende Erhöhung seines Lebens zu [stufenweise] höherer Freiheit.« »Ein bestimmtes Bewußtseyn«, eine bestimmte Bewusstheitsweise von jeweiligen Ist!-Evidenzen, »ist Seyn einer bestimmten Freiheit. Dasjenige nemlich, in Beziehung auf welches die Freiheit frei ist, ist jedesmal der Gegenstand dieses bestimmten Bewußtseyns«, und so sukzessive zu immer höheren, komplexeren Gegenständlichkeits-Welten; und ebenso, daran sich abformend, snach innen<, als Freiheitsgrade des Bewusstseins von Können und Möglichem. „Eine Darlegung der Thatsachen des Bewußtseyns wäre also gleichsam eine Naturgeschichte der Entwicklung dieses Lebens « und dieser seiner, aber im ausgebildeten Bewusstsein schon immer geschehenen, Befreiung zur Bewusstheit auch seiner selbst. ${ }^{27}$

Leibniz hatte die Monaden, als die das Universum repräsentierenden individuellen Seienden, als »lebendige Spiegel« des Seins gefasst - das, was wahr, von ihrem je eigenen »Gesichtspunkt« aus vorstellend.$^{28}$ Fichte zeigt, gegen jede substanzontologische Konzeption, die dem Phänomen der Faktizität dessen, dass Bewusstsein ist und je hier-jetzt situiert wie involviert, nicht gerecht werden kann, allererst die wirkliche Lebendigkeit dieses Lebendigen: mentale Repräsentation des dastehenden außer-mir-Objektiven zu sein, Objektiven einer gemeinsam geteilten Wirklichkeitswelt - der Sachverhaltlichkeiten, Bedeutungen, verstehbaren (zurechenbaren) Handlungen, der Normierungen, Person-Identitäten, und von Vergangenheit wie Geschichts-Horizont; und zugleich, das Dastehende als das dastehend Gesetzte, aktiver involvierter Part, formender Prozess im Gegenüber-Stellen, Gegenständlich-Machen, Reflektieren-Können - und die dazu notwendigen Bilder-Vorstellungen vom Lebendigsein, ohne welche das Denken des Bewusstseins in der Logik der unhinterschreit-

25 In den Thatsachen des Bewußtseyns, in Bezug auf das Bewusstseins-Verhältnis, fungiert die Begrifflichkeit des "Lebens« denn auch vor allem substantivisch, relativ selten in adjektivischer Verwendung.

26 GA II/12, 113.

27 GA II/12, 109 (Hervorh. von mir), 134/29.

$28 \mathrm{Vgl}$. G. W. Leibniz, Principes de la nature et de la grace, fondés en raison, § 3. 
baren Nicht-Einheit sich selbst verborgen bliebe, dem Außen der fremd herrschenden Natur, ihrem Determinismus, anheimgeliefert. ${ }^{29}$ Fichte zeigt die »lebendigen Spiegel«, als die wir existieren, in der Genese all dessen, was erforderlich ist, um die wesenhaften Freiheitsgrade im Geschehen der Bewusstmachung, Bewusstwerdung zu begreifen. Die Philosophische Anthropologie des 20. Jahrhunderts hat hier von der »Positionalität« als der konstitutiven »Daseinsweise [alles] Lebendigen « gesprochen (H. Plessner) - im Hier-Jetzt seiner Situiertheit, in der Faktizität seines Ist, in der Prozessierung seiner Grenze, und als Je-Einzellebendiges. Und hat gesprochen von der »ex-zentrischen Form « dieser Positionalität des `Lebt sich ‘, die durch die Seinspotenz des Bewusstseinsmäßigen entstanden ist. ${ }^{30}$ Fichte, der spätere Fichte, ist eine Theorie, die diese anthropologische Aufgabe beantwortet: sie bereits beantwortet hatte. -

\section{Das Leben des Bewusstseins}

Eine kleine philosophiegeschichtliche Anmerkung mag die Tragweite dieser Fichte'schen Einsichten - dieses Theoriebaus - abschließend erhellen. Der frühe Dilthey, in seinen noch unentschiedenen psychologistischen Anklängen, das Geistige vom Erleben her, dem Vorgang des selbst-eigenen Erlebens aufzubauen, hatte das mit zentrale Theoriestück dabei ebenfalls den, so der Titel, "Tatsachen des Bewußtseins « gewidmet. ${ }^{31}$ Fichte freilich, und mit ihm sein Problembewusstsein, ist darin praktisch nicht präsent. ${ }^{32}$ Fixiert auf die Abgrenzung gegen etwa so etwas wie Helmholtz' > Tat-

29 Die Fichte'sche Konzeption ist darin auch offen furr das, was überhaupt Vor- bzw. Rahmenbedingungen einer anthropologisch nichtbornierten Theorie sind: 1) den evolutionären Zusammenhang mitzuberücksichtigen, dass wir Wesen mit diesem Bewusstsein evolutionär hervorgegangen sind aus nochnichtmenschlichen tierischen Arten (und betreffender tierischer Intelligenz); 2) die ontogenetische Entwicklung des Bewusstseins, die Herausformung seiner vollausgebildeten Struktur beim Kleinkind sowie in sozialen Lernprozessen; 3) die Möglichkeit von Schädigungen und vor allem von Pathologien und geistigen `Krankheiten`. - Im Übrigen geht die transzendentaltheoretische Argumentation in den Thatsachen des Bewußtseyns in Einzelnem durchaus auch parallel mit direkt anthropologischen Sachverhalten. So zum Beispiel, wie unsere Bewusstseinswirklichkeit sich vom basalen Die-Sinne-offen, wo über diese Pforten Wahrnehmungen und Empfindungen unablässig sin uns (hereinströmen - das Bewusstsein dabei nur als das in seinem einzelnen und immer anderen mir-Außen befangenes Ablaufen -, sukzessive - und durchaus auch als Entwicklungsprozess verstanden - zu höheren, formenden und dann im ferneren selbstnormierenden Akten erhebt, verdichtet in der Herauskristallisierung von >Ich/-Gedanken; oder zum Verhältnis von Leiblichkeit und `Seelen`-Vorstellung - die Vorstellung 'Seele` als gewissermaßen nur die intellektuelle Besitzergreifung meines Körpers (und dessen Agierensausstattung), und der, genauer: dessen Gewahrung, wiederum als »Bild« meines (erfahrungsgewordenen) Könnens (vgl. dazu GA II/12, 74-77).

$30 \mathrm{Vgl}$. H. Plessner, Die Stufen des Organischen und der Mensch (1928), in: ders., Gesammelte Schriften, Bd. IV, Frankfurt/M. 1981. Hoch interessant, und trotz allem noch nicht wirklich in seiner theoretischen Tiefe erforscht, ist der Sachverhalt der Beschäftigung Plessners mit Fichte. Sie beginnt mit der frühen Begegnung 1912/13 mit bes. gerade der Wissenschaftslehre 1804 (2. Vortrag) und wurde von ihm mehrfach wieder aufgenommen. Vgl. H. Plessner, »Selbstdarstellung«, in: ders., Gesammelte Schriften, Bd. X, Frankfurt/M. 1985, bes. 308; St. Pietrowicz, Helmuth Plessner, Freiburg/München 1992, 91-118 (bes. $94 \mathrm{f} ., 113-115$ ), $158 \mathrm{f} ., 392-394$.

31 Im entworfenen, aber niemals ganz zu Ende gefuhrten 2. Band seiner Einleitung in die Geisteswissenschaften, dem Kapitel der sog. 'Breslauer Ausarbeitung` (ca. 1880), in: Gesammelte Schriften, Bd. XIX, Göttingen 1982, 58-173.

32 Außer: das II. Buch (»Wissen «) der Bestimmung des Menschen, mit seiner Konzeption der Selbstwahrnehmung - des Selbstbewusstseins in diesem Sinne - als des Fundaments von allem; gerade in diesem 
sachen der Wahrnehmung ${ }^{33}$ fixiert darauf, gegen diese empiristisch-positivistischen Programme, die empiristisch-positivistischen Beerbungen der Transzendentalphilosophie und die sinterventionistische‘ Reformulierung von Realität, Kausalität und Gesetzlichkeit, die irreduzible Erlebnishaftigkeit des Psychischen geltend zu machen, ist genau »Bewußtsein « kaum als Prozess mit eigener Struktur in sich, etwa auch Struktur des Gültigen und des Selbstnormierenden, gesehen, sondern kommt nur als eben Innewerden - verschiedenerlei, aber je subjektiverweise - in den Fokus der Betrachtung; und die »Tatsachen« sind, wie bei den Gegnern, einsinnig Tatsachen für das Bewusstsein, das von ihm als Tatsächlichkeiten phänomenal Erfahrene - das Innewerden als das (als unzweifelhaft-gewiss erfahrene) ses ist ‘ seiner Selbstzuständlichkeit. ${ }^{34}$

Auch wo dies lebensphilosophisch strikter durchgestaltet wurde, war der Preis, dafür den $\mathrm{Be}$ wusstseins-Begriff um so gut wie alle transzendentalphilosophischen Einsichten wieder zu verkürzen: >Bewusstsein` vollends nur als Bewegtheit (in Konsequenz Bergson), oder seine grundsätzliche Insuffizienz (oder doch Winzigkeit/Belanglosigkeit) angesichts der unerschöpflich wogenden Wirklichkeit und der übergroßen Letztfragen des letzten Was-wichtig und Wozu, oder als eben Gebundenheit - Bewusstsein in seiner Leib-Gebundenheit, seiner Triebe-Bedingtheit, in der Gebundenheit an die das wahre Sein erfassende `Seele〈, als der Selbststärke dienliche »Art[en] von Irrtum« (Nietzsche), als alltagsgedrungene Simplifizierungen, als evolutionärerweise Erfolgreiches u. dgl. Bei Fichte dagegen hat der Begriff des »Lebens « weder etwas unkontrolliert Normatives, sachinhaltlich normativ oder theoriearchitektonisch normativ, noch etwas rational Inappellables oder überhaupt Ineffables - so wie schon bei Dilthey kurzerhand statuiert war, "Leben kann nicht vor den Richterstuhl der Vernunft gebracht werden ${ }^{35}{ }^{35}$ Leben vielmehr ist immer schon vor diesem Richterstuhl: ist ein Begriff, den die Vernunftreflexion sich bildet, um das Sein - das radikale Vollzugssein - unserer als Bewusstseins-Wesen zu verstehen. „Leben« ist ein Begriff, um Bewusstsein zu erklären (und das heißt auch: zu erden); ein Begriff, den die Vernunftreflexion hinauswirft, um das Faktum Bewusstsein und dessen ontische Signatur, ontischen Status als je Einzeln-Individuell-Endliches sowie Situiertes, in die Zonen des Verstehbaren zu bringen. ${ }^{36}$ Mittels dieses Begriffs wird rekon-

Fichte-Bezug steht »Tatsache« als mit einem Akzent extremer (Subjekt-) Relativität verbunden. Vgl. ebd., 72-74, $158 \mathrm{f}$. - Zu einem unter ganz anderem Aspekt getätigten Blick auf das Verhältnis Diltheys zu Fichte vgl. auch W. Chr. Zimmerli, "Das Theorem der Tatsachen des Bewußtseins und Diltheys Versuch der Überwindung des transzendentalen Idealismus oder: wie Dilthey den Sack Fichte prügelte und den Esel Reinhold meinte«, in: Dilthey-Jahrbuch, Bd. 3 (1985), 166-190.

33 Hermann v. Helmholtz, Die Thatsachen in der Wahrnehmung, Berlin 1878.

34 Auch später hat Dilthey Fichte kein wirklich tieferes Gewicht zugemessen, hat ihn bekanntlich rein unter die Rubrik Idealismus der Subjektpotenz (»Idealismus der Freiheit «) gefasst, zusammen mit so Unterschiedlichem wie Anaxagoras, christlicher (theistischer) Philosophie, modernem Spiritualismus (einschließlich Bergson) und Pragmatismus. Vgl. Die Typen der Weltanschauung und ihre Ausbildung in den metaphysischen Systemen (1911), in: Gesammelte Schriften, Bd. VIII, Göttingen 1960, 107-112.

35 W. Dilthey, Gesammelte Schriften, Bd. VII, a. a. O., 261. Anspielend offenkundig auf Kants berühmte Gerichtshof-Metapher. - Wie freilich auch in Dilthey etwas, was ein anderer Strang hätte werden können, angelegt war: W. Stegmaier, Philosophie der Fluktuanz. Nietzsche und Dilthey, Göttingen 1992, bes. $162-171$.

36 Der späte Fichte hat sich damit in die Nähe von zumindest einem der anderen Großen des nachkantischen Deutschen Idealismus entwickelt, dem nämlich, der, was die Präsenz im seitherigen Denken betrifft, ihm dauerhaft den Rang abgelaufen hat: Hegel. Ich möchte behaupten, dass genau dies - die Seinsweise des Seienden zu bezeichnen, welchem auch das zugehört, welches denkt (die Seinsweise des Wer des Denkens, der dann exemplarisch von Kierkegaard einseitig eingeklagte sexistierende Denker() - es ist, was das Theoriestiuck des "Lebens", als der "vorausgesetzten oder unmittelbaren Idee", in der Wissenschaft der Logik (1812-16) ausmacht. Aber das wäre noch einmal ein ganz anderes Thema. - In seinen allerletzten Niederschriften hat Fichte noch einmal ganz neu um die Perspektive der höchsten Grundlegung 
struierbar, wie wir - letztlich: in welchen strukturellen Polarisierungen - uns immer schon denken, und denken müssen. »Leben« bei Fichte ist, und a fortiori in jener vorletzten Reflexionsschicht seiner Grundlegungsargumentation, ein (immanenter) Grenz-Begriff der philosophischen Theorie, ein Grenz-Begriff um des Faktums Bewusstsein willen. ${ }^{37}$ Und entsprechend ist bei Fichte mit dem Begriff auch nichts verbunden, was in einer sinnvollen Weise als: Perspektivität und Ähnliches verstanden werden könnte. Was sich von »Leben« her zeigt, ist vielmehr die logische Struktur unseres Bewusstseins - des Kosmos der Bewusstmachung, Kosmos der uns selbst und intersubjektiv wechselseitig verstehbaren Bedeutungen und Gültigkeiten -, in der wir alle - >anthropologisch « - gleich sind, verfassterweise gleich. ${ }^{38}$

und die adäquate Begriffssprache dafür gerungen; zu einem integralen Konzept ist es nicht mehr geworden. Einen Blick darauf, was dies hätte werden können, gibt, als deutliches Dokument dieses Ringens, Fichtes überhaupt letzte abgeschlossene Vorlesung, die Einleitung in die Wissenschaftslehre vom Winter 1813/14, die in einer frappierend heutigen Weise sich als Reflexion auf die Sprache organisiert: die sprachlichen Begriffe - auch die der höchsten Grundlegungsargumentation einer $>$ Wissenschaftslehre $<$-, mittels derer die hochkomplexen transzendentalen Verhältnisse im Bewusstsein gefasst werden können bzw. je schon alltagsweltlich-natürlich kristallisiert sind. Was dabei den Begriff des »Lebens« betrifft, so hat Fichte hier bes. das Problem der Nicht-Einheit noch einmal aufgenommen - »Leben« als Dokument der in der Sprache niedergelegten Weisheit, dass in den höchsten Fragen ein Zusammengehören von Momenten, was sich in der Reflexion in Ursachen-Aporien auflöst, zu denken ist (in Bezug auf Bewusstsein und Denken konkret: die Setzung des Nicht-Selbst, sowie das trotzdem Immer-weiter). Vgl. SW IX, bes. 42-63; für diese letzten Niederschriften insgesamt (in Vorb. für GA II/16) den Vorabdruck in: R. Lauth (Hg.), Ultima inquirenda. J. G. Fichtes letzte Bearbeitungen der Wissenschaftslehre Ende 1813 / Anfang 1814, Stuttgart 2001 [die Einleitungsvorlesungen auch: SW IX, 3-102]. Zum Problem `Leben hier s. auch G. Zöller, »Leben und Wissen. Der Stand der Wissenschaftslehre beim letzten Fichte«, in: E. Fuchs, M. Ivaldo, G. Moretto (Hg.), Der transzendentalphilosophische Zugang zur Wirklichkeit, Stuttgart 2001, 307-330.

37 Das damit eröffnete Potential in ganzer Tiefe zu entfalten blieb freilich bei Fichte, und vollends in der Rezeption dieses transzendentalphilosophischen Erbes, einigermaßen blockiert. Bei Fichte vor allem durch zwei missliche Tendenzen: die Überlagerung mit der (direkt sreligiösen`) Perspektive von scheinbefangenem-vs.-wahrhaftem Leben, d. h. betreffendem Bewusstsein, betreffender Bewusstseinsorientierung; und der (indirekt religiöse) »Sittlichkeits «-Monismus, Panethizismus, der alles Individuelle, und auch das individuelle Leben, nur als Werkzeug des wahrhaften Höheren sieht, als nur von dorther und um deswillen gerechtfertigt. Bei der Rezeption dann hinzu die dazwischengetretene Strömung der konzeptionellen >Lebensphilosophie<, was die drei ebenenmäßig unterschiedenen Zusammenhänge, in denen "Leben« bei Fichte fungiert (Wissenschaft/Leben, Wir-menschliche-»Sinnen«-Wesen, und in der Grundlegungsargumentation der Theorie), hat ineinanderfließen lassen. - Wie das Potential des Fichte'schen Programms prinzipiell wieder verloren wurde, beginnt schon damit, was etwa Schopenhauer, immerhin engagierter Hörer von Fichtes Vorlesungen über die Thatsachen des Bewußtseyns (vgl. GA IV/4, 195-237), daraus mitgenommen hat: der komplexe wechselseitige Prozess von dastehenden strukturellen Tatsachen unseres Mir-ist-bewusst und Leben kommt nur noch als die zwei divergenten Seiten, zwei großen Dimensionen von Idealismus unseres »Vorstellens« und am Eigenleiblichen erfahrener Realität des »Willens « in Thematisierung. Vgl. A. Schopenhauer, Die Welt als Wille und Vorstellung, hg. v. W. v. Löhneysen, Darmstadt 1968, Bd. II, 11-19 / Bd. I, 156-173.

38 Derjenige, in dem das Erbe des Fichte'schen Problembewusstseins mit der Tradition Diltheys zusammengekommen ist: H. Plessner, hat jenes Letztere - in der Formulierung von der primären »Wir-Form des eigenen Ichs « (Gesammelte Schriften, Bd. IV, a. a. O., 377) - zu einem zentralen Theoriestück seiner anthropologischen Theorie gemacht. Gesiegt hat bei ihm am Ende dann aber doch Dilthey. - Zum Fichte-Bezug jenes »Wir«-Theorems in der Entwicklung bei Plessner vgl. St. Pietrowicz, Helmuth Plessner, a. a. $0 ., 434$. 
Jede Forderung nach >Leben! $\iota$, jedes Programm, in dem der Begrifflichkeit von »Leben« und »lebendig« eine zentrale Bedeutung zukommt, ist allemal: Protest, und Korrektiv. Und in diesem an ihrem relativen Ort, in Bezug auf in bestimmtes Gegenüber, wohl meist etwas Berechtigtes, trägt berechtigten Gehalt. Seit der Opposition gegen die formale, mechanistische, kalte ratio der Aufklärung gehören Forderungen und Konzeptionen des >Lebens $\$ denn auch in gewissen regelmäßigen Abständen zum Prozess des modernen Wissenschaftsdenkens. Doch eine konzeptionelle $>$ Lebensphilosophie`, als eine >lebensphilosophische Weise der Argumentation überhaupt und hierin in Frontstellung gegen andere, ist wohl, und bleibt, ein ambivalentes Unternehmen. Das Potential liegt im Begriff »Leben«, nicht in einem epochalen Theorieprogramm >Lebensphilosophie<, wie es sich am Ende des 19. und im ersten Drittel des 20. Jahrhunderts formiert hatte. Da gilt im Gegenteil, dass gerade in dem, wogegen das »Lebens«-Denken der konzeptionellen \Lebensphilosophie، sich aufgerichtet hat, eine noch unausgeschöpfte Sprengkraft liegt. Denn gerade die scheinbar wirklichkeitsnächste Kategorie - „Leben« - ist in hohem Maße ein Theorie-Begriff. Ein Transzendentalphilosoph, Fichte, hat das gesehen.

Rainer Adolphi

Technische Universität Berlin

Institut für Philosophie (H 72)

Straße des 17. Juni 135

D-10623 Berlin

Email: rainer.adolphi@tu-berlin.de 